\title{
Gegen Regelverstöße vorsorgen
}

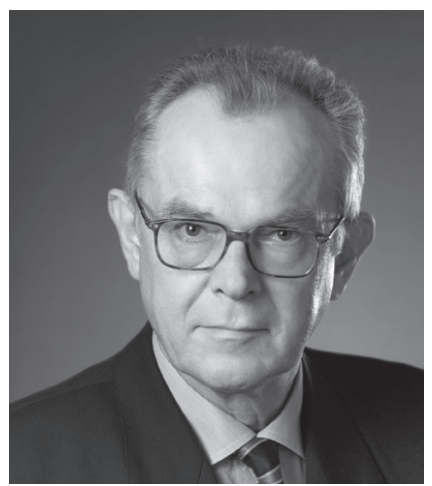

VON CHRISTOPH

\section{KULENKAMPFF}

Rechtsanwalt Christoph Kulenkampff ist Geschäftsführender Partner des Beratungsunternehmens JKW Integrity Services GbR, das sich auf die Abwehr und die unternehmensinterne Aufklärung von Wirtschaftsdelikten und das einschlägige Krisenmanagement spezialisiert hat. Zuvor war er Staatssekretär im hessischen Innenministerium und Generalstaatsanwalt in Hessen. www.jkw-integrity.de

\author{
Verstöße gegen Gesetze, verbandliche oder \\ unternehmensinterne Richtlinien sind bei \\ Organisationen der Sozialwirtschaft aufgrund \\ ihrer öffentlichen Stellung von besonders \\ schmerzhafter Bedeutung. Es ist Aufgabe der \\ Verantwortlichen, gegen solche Regelverletzungen \\ vorzusorgen - und sich rechtzeitig Maßnahmen \\ für »den Fall des Falles" zu überlegen.
}

\begin{abstract}
1 Unter Compliance verstehen wir - heute im Kontext von Wirtschaft und Wirtschaften die Gesamtheit der Maßnahmen, die eine am Wirtschaftsleben teilnehmende Organisation trifft, um das regelkonforme Verhalten seiner Organe und Mitarbeiter im Geschäftsleben zu gewährleisten. Die Regeln, deren Verletzung es zu verhindern gilt, sind konkret etwa die staatlichen Gebote und Verbote, darunter vor allem die sanktionsbewehrten Verbote. Dazu zählen aber auch die selbst eingegangenen Verpflichtungen der Organisation mit Geschäftspartnern sowie ihre internen Regeln zum Geschäftsablauf und nicht zuletzt ihre Unternehmensethik, sofern sie definiert ist. Bei vielen Organisationen sind die Vorstellungen darüber, welche externen (staatlichen) Regelungen sie zu beachten hat, nur lückenhaft. Das Normenspektrum ist auch schier unüberblickbar geworden, und die Verantwortlichen in der Sozialwirtschaft sind gut beraten, sich hierüber für den Tätigkeitsbereich ihrer jeweiligen Organisation zumindest einen ungefähren Überblick zu schaffen.
\end{abstract}

2 Die Sozialwirtschaft steht, was 2. Compliance betrifft, vor besonderen Herausforderungen. Das hat mehrere Ursachen: Typischerweise finanziert sich Sozialwirtschaft nur zu einem (ge- ringeren) Teil aus selbsterwirtschafteten Einnahmen und $\mathrm{zu}$ wesentlichen Teilen aus Zuwendungen der öffentlichen Hand, aber etwa auch aus privaten Spenden. Sozialwirtschaft ist im wesentlichen fremdfinanziert. Insofern kommt es hier in besonderem Maße auf Lauterkeit, Sorgfalt und Ordnungsgemäßheit des allgemeinen Geschäftsgebarens sowie auf Verantwortungsbewusstsein bei der Ressourcenverwendung an. Ferner erbringt Sozialwirtschaft typischerweise personenbezogene Dienstleistungen, und zwar wiederum typischerweise an Personen, die hilfebedürftig sind: etwa Kinder, Jugendliche, Wohnsitzlose, Kranke, Alte, Pflegebedürftige. Die Hilfebedürftigkeit der Klientel lässt Verfehlungen im Umgang mit ihm umso gravierender erscheinen. Schließlich gelten verschärfte Anforderungen an Compliance gerade in der Sozialwirtschaft, weil die meisten dazu gehörenden Organisationen ihren Auftrag aus einer besonderen ethischen oder religiösen Verpflichtung herleiten. Entsprechend streng sind die Maßstäbe, die die Öffentlichkeit an ihr Geschäftsgebaren und vor allem an die Qualität ihrer Dienstleistungen stellt. Immer wieder können wir beobachten, dass Verfehlungen, die im privaten Sektor kaum Erwähnung fänden, im Bereich der Sozialwirtschaft zu massiver öffentlicher Kritik und zu erheblichen 
Reputationsverlusten führen. Insgesamt können wir festhalten, dass in der Sozialwirtschaft Compliance aus guten Gründen einen besonderen Stellenwert haben sollte. Ob die Organisationen der Sozialwirtschaft dies durchgängig auch so sehen, bedürfte einer besonderen Untersuchung; gewisse Zweifel sind jedoch angebracht. Gelegentlich hat man den Eindruck, die Organisationen der Sozialwirtschaft hielten Compliance für entbehrlich, weil sie sich per se "auf der Seite des Guten« sehen.

3 Die für die Sozialwirtschaft denk3. baren Instrumente der Compliance unterscheiden sich grundsätzlich nicht von denjenigen für die allgemeine Privatwirtschaft. Sie können zunächst regulativer Art sein. Hierbei ist an erster Stelle an die Festschreibung der Grundwerte und der konkreten Verhaltensmaximen zu denken, die für die Mitarbeiter der jeweiligen Organisation, übrigens auch die Ehrenamtlichen (!), bei der Erledigung ihrer alltäglichen Aufgaben Geltung haben sollten (»Verhaltenskodex") - vor allem im Umgang mit den Personen, die sich der Einrichtung anvertraut haben bzw. die ihr anvertraut wurden. Auf der Ebene darunter kommen Regeln zu den einzelnen Geschäfts- abläufen in der jeweiligen Organisation in Betracht, beispielsweise zum Verfahren bei der Aufnahme in Einrichtungen, zum Einkauf, auch zum Bareinkauf, und zur Auftragsvergabe etwa beim Bau und der Bauunterhaltung. Eines ist aber bei der Schaffung interner Regeln stets zu beachten: Nichts ist mit Blick auf Compliance kontraproduktiver als Überregulierung!

4 Nicht weniger wichtig als eine - Grundausstattung mit Verhaltensund Verfahrensregeln ist eine in sich stimmige Aufbauorganisation, welche die Zuständigkeit und damit auch Verantwortlichkeit eines jeden Mitarbeiters exakt festlegt, die Hierarchie in der Organisation darstellt und die bestimmt, wer wen worin bei Abwesenheit vertritt. Häufig begünstigt gerade das Fehlen einer stringenten Aufbauorganisation mit einem »Geschäftsverteilungsplan « und einem Organigramm Regelverstöße von Mitarbeitern, weil sie sich durch Unklarheiten hinsichtlich der Verantwortlichkeit für einzelne Geschäftsvorgänge geschützt fühlen.

5icht zuletzt gehört zu den übli2. chen, auch für die Sozialwirtschaft einschlägigen Compliance-Maßnahmen eine funktionsorientierte Personalauswahl und Personaleinsatz: Wer gehört nach seiner fachlichen und vor allem persönlichen Qualifikation auf welchen Arbeitsplatz? Die Antwort hierauf setzt natürlich eine genaue Beschreibung der Anforderungen für den jeweiligen Arbeitsplatz (»Anforderungsprofil«) voraus.

6. Noch einen Satz für den Fall, dass »das Kind in den Brunnen gefallen ist ", also in den eigenen Reihen gegen die Regeln verstoßen wurde und zudem noch die Öffentlichkeit davon weiß oder $\mathrm{zu}$ wissen droht. Am gravierendsten wiegt hier in vielen Fällen nicht der Regelverstoß für sich, sondern der Umgang mit ihm nach innen und gegenüber der Öffentlichkeit. Der Fall des ehemaligen Bundespräsidenten Wulff ist ein Beispiel für extrem misslungenes Krisenmanagement. Gerade die Organisationen der Sozialwirtschaft mit ihrer besonders sensiblen Wahrnehmung durch die Öffentlichkeit sollten Krisenmanagement präventiv implementieren, denn sie haben viel an gutem Ruf zu verlieren. Die rückhaltlose "Flucht nach vorne" wird in der Regel angezeigt sein.

\title{
Instrumentenreform bereits berücksichtigt.
}

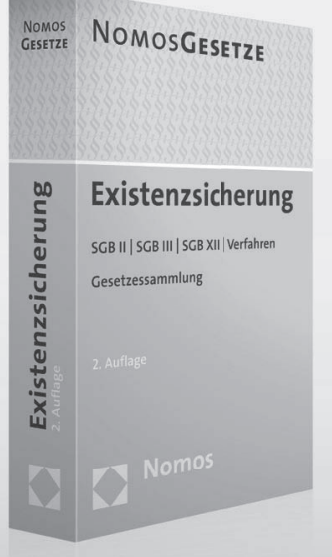

\author{
Existenzsicherung \\ SGB II | SGB III | SGB XII | Verfahren \\ Gesetzessammlung \\ 2. Auflage 2012, 799 S., brosch., 9,90€ \\ ISBN 978-3-8329-7346-9
}

Die Textsammlung enthält alle relevanten, existenzsichernden Grundsicherungsnormen des SGB II, SGB III und XII, die wesentlichen Verordnungen sowie Verfahrensregelungen.

Weitere Informationen: www.nomos-shop.de/14543

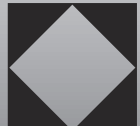

Nomos 